\title{
Impact of Antibiotic Prophylaxis on the Conjunctival Flora of Patients Receiving Intravitreal Antivascular Endothelial Growth Factor Injections
}

\author{
Maria Picoto Passarinho ${ }^{a}$ Joana R. Portelinha ${ }^{a}$ Teresa J. Pacheco ${ }^{b}$ \\ António E. Rodrigues ${ }^{a}$ Teresa P. Marques ${ }^{c}$ Fernanda F. Vaz ${ }^{a}$ \\ ${ }^{a}$ Ophthalmology Department, ${ }^{b}$ Clinical Pathology Department, and ${ }^{\mathrm{C}}$ Laboratory \\ and Clinical Medicine Department, Egas Moniz Hospital (H.E.M.), Centro Hospitalar de \\ Lisboa Ocidental (C.H.L.O.), Faculdade de Ciências Médicas, CEDOC, Universidade \\ Nova de Lisboa, Lisbon, Portugal
}

Key Words

Prophylaxis · Antibiotic resistance · Conjunctival flora $\cdot$ Intravitreal injections · Antivascular endothelial growth factor

\section{Abstract}

Purpose: To characterize the conjunctival flora of patients receiving antibiotics before intravitreal (IVT) antivascular endothelial growth factor injections. Methods: We studied 31 eyes of 30 patients with neovascular age-related macular degeneration scheduled to receive IVT antivascular endothelial growth factor injections. The patients were all given a preinjection of topical ofloxacin 3 days before the injections. Conjunctival cultures from the injection eye were collected before antibiotic prophylaxis (sample A) and on the injection day, both before (sample B) and after (sample C) povidone-iodine (PVI) application. Results: Culture positivity was significantly different between samples $A(50 \%)$ and $C(4.5 \% ; p<0.001)$ as well as samples $B(30.4 \%)$ and $C(4.5 \% ; p=0.015)$, but not between samples $A$ and $B$. Positive cultures were mainly coagulase-negative staphylococci (CNS; $57 \%$ of sample $A, 62.5 \%$ of sample $B$ and $100 \%$ of sample C) susceptible to vancomycin (100\%), moxifloxacin (90\%), levofloxacin (70\%) and ofloxacin (60\%). Conclusion: In our study, most of the bacteria isolated were CNS. PVI application but not antibiotics reduced culture positivity. 
Picoto Passarinho et al.: Impact of Antibiotic Prophylaxis on the Conjunctival Flora of Patients Receiving Intravitreal Antivascular Endothelial Growth Factor Injections

\section{Introduction}

Intravitreal (IVT) injections are the most common treatment modality for several retinal pathologies, namely macular edema, diabetic retinopathy and age-related macular degeneration (ARMD) [1]. In 2009, over 1,270,000 injections were carried out in the USA [2]. The incidence rate of endophthalmitis following this procedure is 0.019 to $1.4 \%$ [3], meaning that approximately 1 in every 1,000-5,000 injections results in endophthalmitis [2]. Despite the relatively low risk of this entity to occur, the number of cases referred to injection has progressively increased in the last years, and the procedure has become common practice. On the other hand, endophthalmitis, albeit rare, generally produces devastating effects.

The etiology of post-IVT infectious endophthalmitis is based mostly on two mechanisms: direct inoculation at the time of the injection or inoculation a posteriori through needle insertion in the eye, particularly in cases of vitreous incarceration.

The correlation between bacteria on the eye surface and the risk of developing endophthalmitis remains to be proven. However, it is believed that most bacterial isolates in endophthalmitic patients after IVT injection possibly arise from the bacterial flora on the eye surface [4].

Consequently, prophylactic measures for minimizing the risk of endophthalmitis have been outlined. One of the most accepted prophylactic measures is to prepare the injection site with povidone-iodine (PVI) as this is the only proven method for decreasing the risk of endophthalmitis following IVT surgeries [5].

The use of topical antibiotics prior to IVT remains controversial. Halachmi-Eyal et al. [6] concluded that endophthalmitis prophylaxis with preinjection of antibiotics does not reduce the endophthalmic rate, nor does it decrease the bacterial count any more than the use of PVI alone [7]. According to Bhavsar et al. [8, 9], the benefit of preinjection of topical antibiotics is not demonstrated. Besides, increased bacterial resistance to preinjection of antibiotics, particularly in the context of monthly repeated injections, has been described [2].

As for the use of post-IVT antibiotics, according to a study by Bhatt et al. [10], the rate of endophthalmitis is equivalent with or without antibiotics.

Ta et al. [11], on the other hand, suggest that the combination of PVI and antibiotics may trigger a reduction of bacterial cultures on the eye surface.

These studies' conflicting findings indicate that the efficacy of antibiotic prophylaxis in preventing endophthalmitis after intraocular injection is still unknown. Consequently, and considering the devastating impact of endophthalmitis, it is still deemed good practice to administer a topical antibiotic (quinolone) prior to intraocular procedures.

The main objective of this study is to characterize the conjunctival flora of patients who received prophylactic topical antibiotics before IVT antivascular endothelial growth factor injection for neovascular ARMD at three different moments: (A) before antibiotic prophylaxis, (B) after antibiotic prophylaxis and (C) after PVI application. The secondary end point was to analyze antimicrobial susceptibility patterns of the bacteria isolated in the lower conjunctival sac.

\section{Materials and Methods}

This study is a nonrandomized case series that included patients consecutively diagnosed with neovascular ARMD and referred for anti-angiogenic therapy between June 1 and November 30, 2012. Patients were excluded from participation if they had previously been treated with IVT injections, were taking topical or systemic antibiotics, had an active ocular 
infection, wore contact lenses or were submitted to other ocular procedures such as laser or ocular surgery.

Institutional Review Board (IRB)/Ethics Committee approval was obtained for this study. It adhered to the tenets of the Declaration of Helsinki and all patients gave their informed consent to participate.

The antiangiogenic therapy used was ranibizumab $0.5 \mathrm{mg}$ (Lucentis $10 \mathrm{mg} / \mathrm{ml}$; Novartis Pharma). All patients were instructed to instill 1 drop of topical ofloxacin (Floxedol; Edol, Lindaa-Velha, Portugal) into their treated eye 4 times a day, starting 3 days before IVT injection.

Cultures were obtained from the inferior forniceal conjunctiva using Eurotubo collection swabs at the three different moments: on the first visit prior to antibiotic application, on the day of the procedure before PVI instillation and $90 \mathrm{~s}$ after PVI disinfection. Extreme attention was paid with the swab, avoiding lids and lashes adjacent to the culture site. The collections were repeated after every new injection.

IVT injections were performed in the operating room in a sterile setting by 3 retina surgeons. Before injection, a culture of the injection eye was collected from the inferior forniceal conjunctiva, 1 drop of hydrochloride of oxibuprocaine (Anestocil; Edol) was instilled along with 1 drop of tropicamide (Tropicil Top $10 \mathrm{mg} / \mathrm{ml}$; Edol), 1 drop of phenylephrine (Davinefrina $100 \mathrm{mg} / \mathrm{ml}$, Davi II; Barcarena, Portugal) and another ofloxacin. The eye was then allowed to rest for $10 \mathrm{~min}$. During this period, the treatment drug was extracted into the injection syringe before the procedure with strict adherence to sterile technique. Next, a drop of PVI 5\% (Betadine $50 \mathrm{mg} / \mathrm{ml}$ cutaneous solution; Meda Pharma, Lisbon, Portugal) was instilled into the inferior forniceal conjunctiva of the injection eye, and the patient was instructed to close his eye. The eyelids and lashes were then painted with PVI 10\% swabs, and after $3 \mathrm{~min}$, a sterile lid speculum was placed by touching the eyelids with a sterile cottontipped applicator. A second culture of the injection eye was collected in a similar fashion as earlier cultures.

Immediately after culture collection, the injection was performed in the inferotemporal quadrant at a distance of 3.5 to $4.0 \mathrm{~mm}$ from the superior temporal limbus (according to lens status), taking care to avoid contact between the lids and lashes and the needle. After retraction of the needle, the injection site was immediately covered with a sterile, cotton-tipped applicator to minimize bleeding and possible backflow of medication through the puncture site. Finally, ofloxacin eyedrops were administered.

The samples were put in Columbia blood agar with 5\% sheep blood (Biomérieux, Paris, France), PolyViteX chocolate agar (PVX; Biomérieux) and Sabouraud medium (Biomérieux) and incubated at $37^{\circ} \mathrm{C}$ for $48 \mathrm{~h}$ (if a sterile culture, it was incubated for additional $24 \mathrm{~h}$ ), having established that any bacteria or yeast isolates would be considered.

Identification was provided using the Vitek2 system (Biomérieux) or other manual methods used in the laboratory.

Antimicrobial susceptibility patterns were determined by the genus and species of isolated microorganisms using the cards of the automated Vitek2 system. Where justified (for the three quinolones studied), the Etest was applied (Biomérieux; Clinical and Laboratory Standards Institute, CLSI, consensus tables).

On positive cultures, susceptibility was tested for fusidic acid, ofloxacin, levofloxacin, moxifloxacin, vancomycin, gentamicin, tetracycline, tobramycin, TMP/SMX, clindamycin, erithromycin, fosfomycin, linezolid, mupirocin, nitrofurantoin, oxacillin, penicillin G, rifampicin, teicoplanin and tigecycline.

All isolated bacteria were necessarily tested for three quinolones, ofloxacin, levofloxacin and moxifloxacin, and the relevant minimum inhibitory concentrations were assayed. When the cards of the Vitek2 automated system did not include these antimicrobials, the minimum inhibitory concentrations were assayed using the Etest method (Biomérieux). 
Fig. 1. Proportion of positive cultures at moments $\mathrm{A}, \mathrm{B}$ and $\mathrm{C}$.
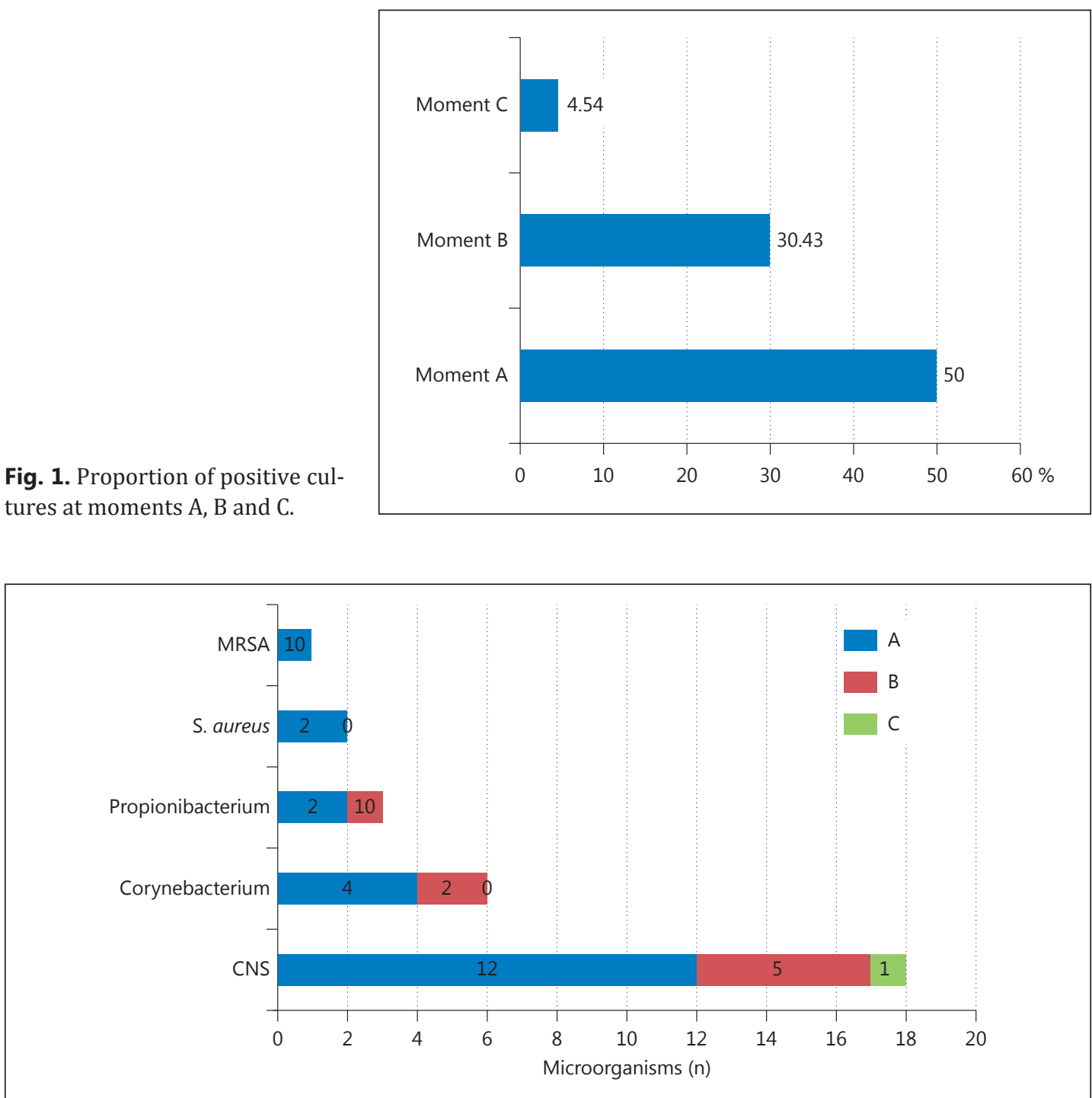

Fig. 2. Microorganisms isolated at moments A, B and C.

The cases were stratified in different subgroups by gender, age, associated pathology, which may influence local immunity, and number of injections performed. Each group was submitted to a microorganism profile and resistance study.

Statistical analysis was performed using the Fisher's exact test and Pearson coefficient. $\mathrm{p} \leq 0.05$ was considered significant for the purpose of drawing up the conclusions.

\section{Results}

This prospective study involved 30 patients (31 eyes) with a mean age of $78.57 \pm 7.15$ (distribution 63-90). Twelve were males (40\%) and 18 females (60\%), and $20 \%$ of all patients were diabetic. The average number of injections per patient was 2.61 .

The number of positive cultures was $15(50.0 \%)$ at moment A, 7 (30.43\%) at moment B and $1(4.54 \%)$ at moment C. A statistically significant difference was observed in the rate of 


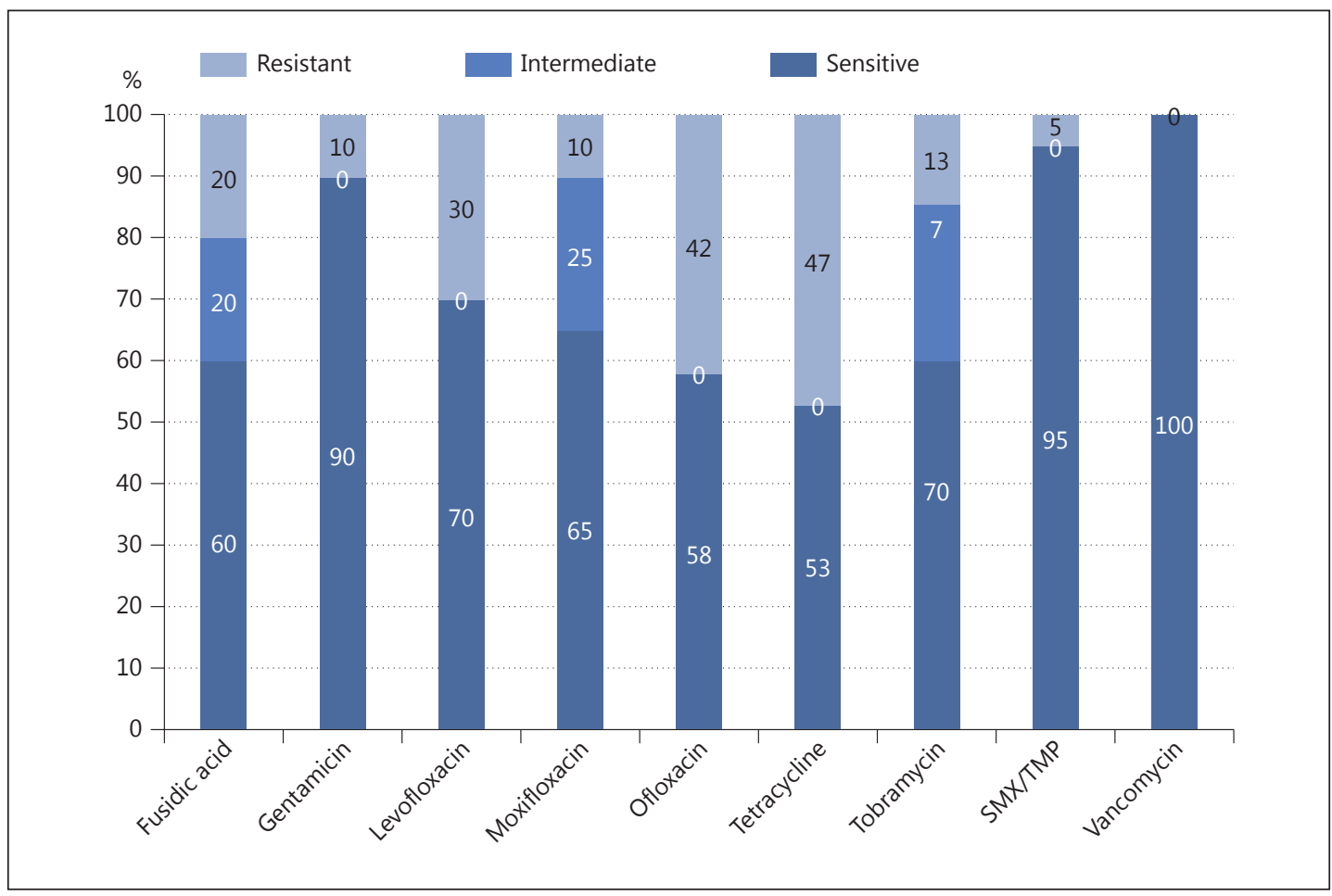

Fig. 3. Antibiotic sensitivity.

Table 1. Microorganisms detected in patients with co-colonization

\begin{tabular}{lllll}
\hline & \multicolumn{3}{l}{ Microorganisms } & \\
\cline { 2 - 5 } & CNS & S. aureus & $\begin{array}{l}\text { Coryne- } \\
\text { bacterium }\end{array}$ & $\begin{array}{l}\text { Propioni- } \\
\text { bacterium }\end{array}$ \\
\hline Patient 1 & 1 & 1 & 0 & 0 \\
Patient 2 & 0 & 0 & 1 & 1 \\
Patient 3 & 2 & 0 & 1 & 0 \\
Patient 4 & 1 & 0 & 0 & 1 \\
Patient 5 & 1 & 0 & 1 & 0 \\
Patient 6 & 1 & 1 & 0 & 0 \\
Patient 7 & 1 & 0 & 1 & 0 \\
\hline
\end{tabular}

positive cultures between moments $A$ and $C$ and moments $B$ and $C(p<0.001$ and $p=0.015$, respectively, Fisher's exact test). However, the difference in culture positivity between moments A and B ( $p=0.197$ ) was not statistically significant (fig. 1).

At moment A, 21 microorganisms were isolated: 12 coagulase-negative staphylococci (CNS; 60\%), the most common ones being Staphylococcus epidermidis (57\%); 4 Corynebacterium sp. (19\%); 2 Propionibacterium sp. (9\%); 2 S. aureus (9\%), and 1 MRSA (4.7\%). At moment B, 8 microorganisms were isolated: 5 CNS (62.5\%), 2 Corynebacterium sp. (25.0\%) and 1 Propionibacterium sp. (12.5\%). At moment C, 1 CNS was isolated (fig. 2).

Between moments A and B, when patients were submitted to antibiotic therapy, 5 eyes (16.1\%) developed colonies of new microorganisms. Of the 5 CNS isolated, $3(60 \%)$ were sensitive to ofloxacin, while $2(40 \%)$ were resistant to this antibiotic, one of them having developed resistance during prophylaxis before the second injection. Between moments $\mathrm{B}$ and C, after PVI application, only 1 CNS isolate remained. 
Fig. 4. Fluoroquinolone resistance profile.

Fig. 5. Proportion of CNS resistant to multiple antibiotics.
Picoto Passarinho et al.: Impact of Antibiotic Prophylaxis on the Conjunctival Flora of Patients Receiving Intravitreal Antivascular Endothelial Growth Factor Injections
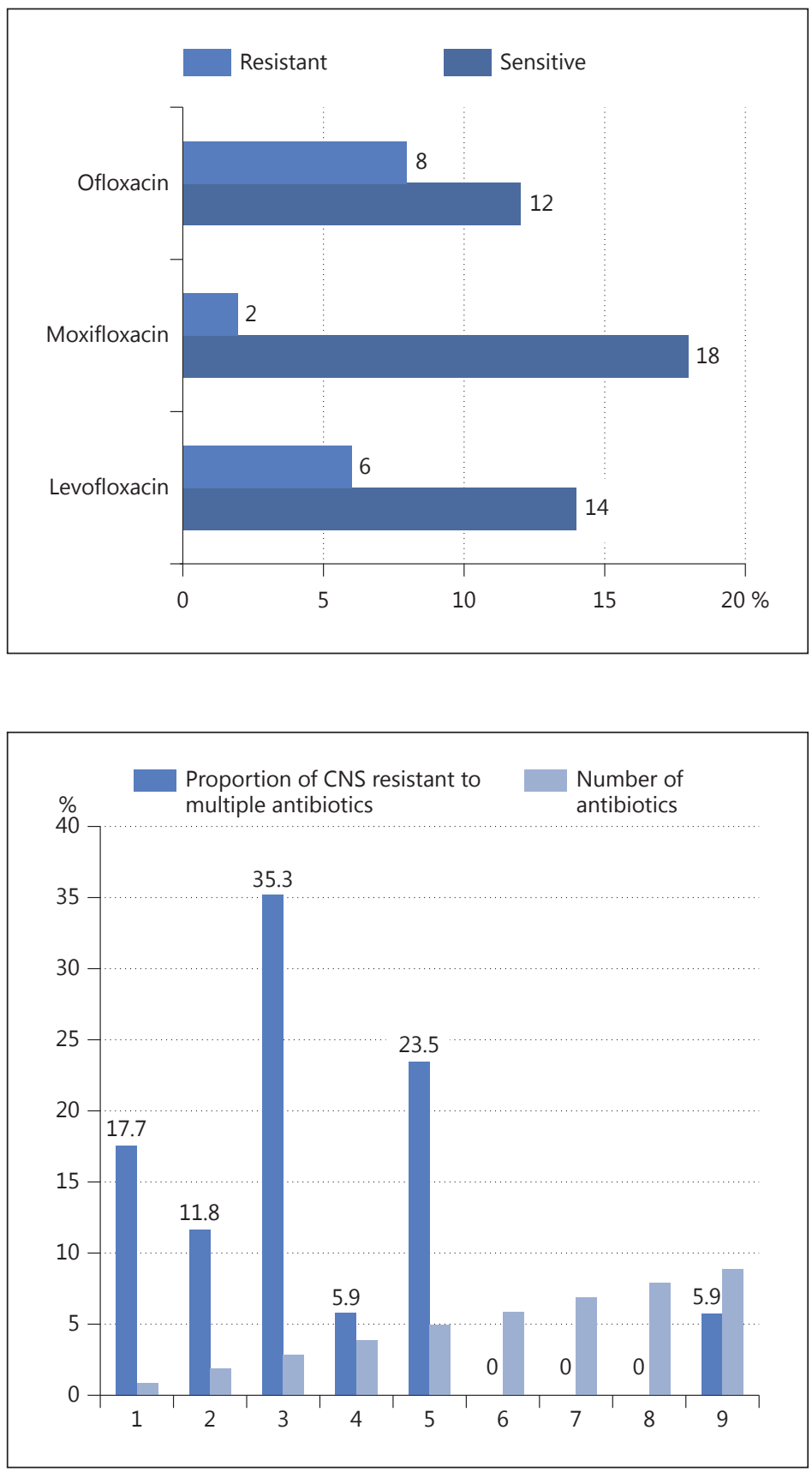

More than 1 microorganism was identified in $7(22,58 \%)$ of the study eyes, $6(85,7 \%)$ at moment A (eyes 1-6) and 1 (14,29\%) at moment B after the second injection (eye 7) (table 1).

Culture positivity was compared at the three moments between diabetic and nondiabetic patients. Only at moment $B$, a statistically significant difference was found $(\mathrm{p}=0.01)$ between these two patient groups, and the number of microorganisms in the diabetic group was higher $(63 \%)$ than in the nondiabetic group (9\%).

When the gender subgroups were analyzed, the rate of positive cultures was found to be higher in males (67\%) compared to females (41.0\%) in all samples and at all moments, although this was not statistically significant $(\mathrm{p}=0.189)$. 
Table 2. Resistance profile of CNS at moments A, B and C to the different families of the antibiotics analyzed

\begin{tabular}{llllll}
\hline CNS & Moment & Tetracycline & Aminoglycosides & Fluoroquinolones & Sulfonamides \\
\hline 1 & $\mathrm{~A}$ & $\mathrm{~S}$ & $\mathrm{R}$ & $\mathrm{R}$ & $\mathrm{R}$ \\
2 & $\mathrm{~A}$ & $\mathrm{~S}$ & $\mathrm{~S}$ & $\mathrm{R}$ & $\mathrm{S}$ \\
3 & $\mathrm{~B}$ & $\mathrm{~S}$ & $\mathrm{~S}$ & $\mathrm{~S}$ & $\mathrm{~S}$ \\
4 & $\mathrm{~B}$ & $\mathrm{~S}$ & $\mathrm{~S}$ & $\mathrm{~S}$ & $\mathrm{~S}$ \\
5 & $\mathrm{~A}$ & $\mathrm{R}$ & $\mathrm{S}$ & $\mathrm{R}$ & $\mathrm{S}$ \\
6 & $\mathrm{~A}$ & $\mathrm{R}$ & $\mathrm{S}$ & $\mathrm{S}$ & $\mathrm{S}$ \\
7 & $\mathrm{~B}$ & $\mathrm{R}$ & $\mathrm{S}$ & $\mathrm{S}$ & $\mathrm{S}$ \\
8 & $\mathrm{~B}$ & $\mathrm{R}$ & $\mathrm{S}$ & $\mathrm{R}$ & $\mathrm{S}$ \\
9 & $\mathrm{~B}$ & $\mathrm{~S}$ & $\mathrm{~S}$ & $\mathrm{~S}$ & $\mathrm{~S}$ \\
10 & $\mathrm{~A}$ & $\mathrm{R}$ & $\mathrm{S}$ & $\mathrm{S}$ & $\mathrm{S}$ \\
11 & $\mathrm{~A}$ & $\mathrm{R}$ & $\mathrm{S}$ & $\mathrm{S}$ & $\mathrm{S}$ \\
12 & $\mathrm{~B}$ & $\mathrm{~S}$ & $\mathrm{~S}$ & $\mathrm{R}$ & $\mathrm{S}$ \\
13 & $\mathrm{~A}$ & $\mathrm{R}$ & $\mathrm{S}$ & $\mathrm{S}$ & $\mathrm{S}$ \\
14 & $\mathrm{~A}$ & $\mathrm{R}$ & $\mathrm{S}$ & $\mathrm{S}$ & $\mathrm{S}$ \\
15 & $\mathrm{~A}$ & $\mathrm{~S}$ & $\mathrm{~S}$ & $\mathrm{~S}$ & $\mathrm{~S}$ \\
16 & $\mathrm{~A}$ & $\mathrm{R}$ & $\mathrm{S}$ & $\mathrm{S}$ & $\mathrm{S}$ \\
17 & $\mathrm{~A}$ & $\mathrm{~S}$ & $\mathrm{~S}$ & $\mathrm{R}$ & $\mathrm{S}$ \\
18 & $\mathrm{~A}$ & $\mathrm{~S}$ & $\mathrm{R}$ & $\mathrm{R}$ & $\mathrm{S}$ \\
\hline
\end{tabular}

$\mathrm{S}=$ Sensitive, $\mathrm{R}=$ resistant

The correlation between positive cultures seen at the three moments as well as patient age were assessed, but no correlation could be found between these variables (Pearson correlation, $\mathrm{p}=0.64$ at moment $\mathrm{A}, \mathrm{p}=0.65$ at moment $\mathrm{B}$ and $\mathrm{p}=0.68$ at moment $\mathrm{C}$ ).

Figure 3 illustrates antibiotic susceptibility of the isolated microorganisms in the lower conjunctival sac. They were all sensitive to vancomycin. The following were the resistance profiles found for the main antibiotics used in our clinical practice: $10 \%$ for gentamicin; $30 \%$ for levofloxacin; $10 \%$ for moxifloxacin; $40 \%$ for ofloxacin, $47.4 \%$ for tetracycline; $13.3 \%$ for tobramycin, and $5.3 \%$ for TMP/SMX.

As for the resistance profile of fluoroquinolones, the number of resistant isolates of ofloxacin was higher $(8,40 \%)$ than that of levofloxacin $(6,30 \%)$ and moxifloxacin $(2,10 \%)$. This fourth-generation fluoroquinolone was found to be more sensitive than third-generation fluoroquinolones (fig. 4).

Two $S$. aureus strains were isolated in 2 patients at moment A. Both were sensitive to the antibiotics tested (fusidic acid, gentamicin, ofloxacin, oxacillin, levofloxacin and moxifloxacin). An MRSA was isolated, also at moment A, which was sensitive to vancomycin, tetracycline, tobramycin, TMP/SMX and resistant to the three fluoroquinolones studied (fig. 5).

Analyzing the families of the antibiotics, $5.6 \%$ of the CNS were resistant to three families of antibiotics and $16.7 \%$ were resistant to two families. The families with the highest resistance rates were the following (in decreasing order): tetracycline [9 CNS (50\%) were resistant]; fluoroquinolones [1 CNS (5\%) was resistant to the three fluoroquinolones studied, 2 CNS were resistant only to ofloxacin and 4 CNS $(22,2 \%)$ to ofloxacin and levofloxacin]; aminoglycosides [2 CNS (11\%) were resistant to gentamicin and tobramycin] and SMX/TMP [1 CNS (5\%) was resistant] (table 2).

The profile of resistance to the three fluoroquinolones (levofloxacin, ofloxacin and moxifloxacin) was correlated with age and number of injections. No statistically significant correlation was found between the variables (Pearson coefficient, $p>0.05$ ).

In this study, the mycological examination of all samples was negative in $100 \%$ of the cases. 
Picoto Passarinho et al.: Impact of Antibiotic Prophylaxis on the Conjunctival Flora of Patients Receiving Intravitreal Antivascular Endothelial Growth Factor Injections

\section{Discussion}

Current therapeutic management of exudative ARMD requires IVT injections at approximately a monthly rate and often over a long period of time. Endophthalmitis is the procedure's worst complication, the incidence rate of which is variable in the literature. More recent studies estimate the risk at around $0.02-0.16 \%$ per injection [12].

The bacterial conjunctival flora enters the eye possibly during or after IVT. For more accurate prophylaxis in this context, the number of microorganisms at the injection site and during the injection period must be reduced.

In 2004, guidelines for reducing the risk of post-IVT endophthalmitis were published, which postulate that the lower conjunctival sac and the eyelashes must be topically disinfected with PVI, the speculum should be used for opening the eye and the needle must be prevented from coming into contact with other eye surfaces than the injection site [13].

Currently, there is no agreement on the topical antibiotics that should be used either before or after the injections. Recent studies found that these are not an advantage in terms of reducing the rate of endophthalmitis [8, 9]. However, since there is a rationale for their use (eliminating the bacterial conjunctival flora and reducing the pathogenicity of the surviving bacteria) [14, 15], it is not surprising that according to the Green-Simms study [16], around $2 / 3$ of the retinal specialists use them for IVT endophthalmitis prophylaxis.

The choice of the antibiotic, regardless of when it is used, must focus on the microorganisms in the conjunctival flora, which are most likely to cause endophthalmitis.

The microorganisms most frequently found in our study were CNS (corresponding to $57 \%$ of the isolates at moment $\mathrm{A}, 62.5 \%$ at moment $\mathrm{B}$ and to the microorganisms identified at moment $\mathrm{C}$ ). This finding is consistent with previous studies [15, 17]. CNS are involved in approximately $70 \%$ of the cases of postoperative endophthalmitis [18]. Of the CNS isolated in the Endophthalmitis Vitrectomy Study [19], the strain responsible for endophthalmitis was S. epidermidis (81.9\%). In our series, this microorganism was identified in $55 \%$ of the cases at moment $\mathrm{A}$. Consequently, this study supports the hypothesis that the conjunctival flora is the main source of infection in endophthalmitis.

As for the sensitivity patterns of the conjunctival flora, we see that most microorganisms are sensitive to fusidic acid, gentamicin, levofloxacin, moxifloxacin, tobramycin and TMP/ SMX. Sensitivity to ofloxacin is found in only $55 \%$ of the strains. None of the microorganisms are resistant to vancomycin. Multiple resistance (resistance to $\geq 2$ antibiotics) was found in $22.3 \%$ of the CNS. Gentamycin, TMP/SMX and vancomycin cover the largest number of strains analyzed.

In our study, through the comparative positivity analysis of the samples (between moment A, initial visit; moment B, after prophylaxis with antibiotics, and moment $\mathrm{C}$, after disinfection with PVI), a statistically significant difference was found only between moments $A$ and $C$ as well as $B$ and $C$. This result matches the findings in the recent literature, suggesting the following: absence of benefits in the use of preinjection of antibiotics to reduce the rate of endophthalmitis and bacterial count [6], disinfection with PVI is the only method with proven efficacy to reduce the rate of endophthalmitis after eye surgery [5]; the use of topical antibiotics does not grant additional benefit to the isolated use of PVI for reducing the conjunctival flora bacteria, and the use of topical PVI is an efficient monotherapy for preventing post-IVT injection endophthalmitis [20].

Furthermore, at moment $\mathrm{B}$, the microorganisms found probably came from recolonization with new microorganisms, since they were not seen at moment A. Of the $5 \mathrm{CNS}$ isolated, 3 were sensitive to ofloxacin and 2 resistant to this antibiotic. CNS sensitive to the antibiotic applied for prophylactic purposes may arise from the incorrect use of this antibiotic by the same patient. The two CNS resistant to ofloxacin are partly attributable to the excessive use 
of antibiotics in current clinical practice, particularly in one of the patients, in whom the CNS only appeared during the second injection. Besides CNS, two Corynebacterium sp. and one Propionibacterium sp. were found, which, although ubiquitous microorganisms in the conjunctival flora, are pathogenic agents in immunocompromised individuals. This must be taken into account, considering the advanced age and the multiple pathologies presented by these patients.

At moment A, an MRSA was detected, which later on was not isolated in $\mathrm{B}$ and $\mathrm{C}$, although this microorganism is resistant to the antibiotics used for prophylaxis.

In 7 of the patients studied, the conjunctival flora was colonized by different bacteria. Six of these patients had been co-colonized at moment A, 2 of them with CNS and S. aureus concomitantly. However, the topical antibiotic applied helped to inhibit growth. In one of the 7 patients, a CNS and a Corynebacterium were isolated only at moment B, probably arising from recolonization.

Epidemiological factors correlated with endophthalmitis have been described by several authors, particularly in the context of cataract surgery. According to Hatch et al. [21], endophthalmitis occurring after cataract surgery is more frequent in male patients and at older age. We do know that ARMD is more frequent in patients at or above the age of 75 years and in females, as shown in our sample (the average age was $78.57 \pm 7.15$ years, and $60 \%$ were women). In the study by Moss [20], age was not correlated with the number of positive cultures, the rate of which was higher in men.

In our study, we have assessed the correlation between patient age and positive cultures (Pearson correlation) and compared the cultural findings between men and women. We have not found any correlation between positive samples and age $(\mathrm{p}>0.05)$, nor any statistically significant difference between men and women $(p=0.24)$.

We have also compared the number of positive cultures in diabetic and nondiabetic patients. We have found that after applying antibiotics, the proportion of positive cultures was higher in diabetic patients (63 vs. 9\%, $\mathrm{p}=0.01$ ). According to several studies of endophthalmitis, in the scope of cataract surgery and vitrectomy, diabetes mellitus was established as a risk factor $[22,23]$. This finding may be connected to the fact that diabetic patients have compromised immunity and subsequent delayed healing and bacterial clearance [24]. In the Endophthalmitis Vitrectomy Study [19], diabetic patients showed more virulent microorganisms, a higher proportion of Gram negatives and less probability of presenting negative cultures.

We also found that the profile of resistance to the three fluoroquinolones (levofloxacin, ofloxacin and moxifloxacin) was correlated with the number of injections, indicating that resistance increased with this number. However, as for the study constraints, the small number of patients and the fact that the exudates were collected before the injection did not allow us to analyze the changes in the flora during the post-injection period.

In conclusion, CNS were the most frequently isolated microorganisms from the conjunctival flora before IVT injections. Although $40 \%$ are resistant to ofloxacin ( $28 \%$ at moment B) and $22 \%$ are resistant to multiple antibiotics, all isolated strains are sensitive to vancomycin. Thus, ofloxacin does not seem to be the best choice for endophthalmitis prophylaxis. Most microorganisms were sensitive to fusidic acid, gentamicin, levofloxacin, moxifloxacin, tobramycin and TMP/SMX.

In contrast to PVI application, administration of antibiotics before IVT injection did not reduce the number of recolonizations with new bacteria. In short, we studied the conjunctival flora of 30 patients receiving antibiotic before IVT antivascular endothelial growth factor injections. The bacterial isolates were predominantly positive for CNS. Antibiotic prophylaxis with ofloxacin did not reduce the number of positive cultures as opposed to PVI application. 
Ophthalmologica
Ophthalmologica Extra 2014;1:9-18

DOI: $10.1159 / 000358223$

(2)

C) 2014 S. Karger AG, Basel www.karger.com/opx

Picoto Passarinho et al.: Impact of Antibiotic Prophylaxis on the Conjunctival Flora of Patients Receiving Intravitreal Antivascular Endothelial Growth Factor Injections

\section{Disclosure Statement}

No conflicting relationship exists for any author.

\section{References}

$>1$ Moss JM, Stanislo SR, Ta C: Antibiotic susceptibility patterns of ocular bacterial flora in patients undergoing intravitreal injections. Ophthalmology 2010;117:2141-2145.

2 Harry WF, Charles CW, Philip JR: Antiseptics vs antibiotics for intravitreal injections. Subspeciality Day 2011: Retina. http://one.aao.org/annual-meeting-video/antiseptics-vs-antibiotics-intravitreal-injections-2.

-3 Cheung CS, Wong AW, Lui A, Kertes PJ, Devenyi RG, Lam WC: Incidence of endophthalmitis and use of antibiotic prophylaxis after intravitreal injections. Ophthalmology 2012;119:1609-1614.

-4 Moshirfar M, Feiz V, Vitale AT, Wegelin JA, Basavanthappa S, Wolsey DH: Endophthalmitis after uncomplicated cataract surgery with the use of fourth-generation fluoroquinolones: a retrospective observational case series. Ophthalmology 2007;114:686-691.

5 Speaker MG, Menikoff JA: Prophylaxis of endophthalmitis with topical povidone-iodine. Ophthalmology 1991; 98:1769-1775.

6 Halachmi-Eyal O, Lang Y, Keness Y, Miron D: Preoperative topical moxifloxacin 0,5\% and povidone-iodine $5,0 \%$ versus povidone-iodine $5,0 \%$ alone to reduce bacterial colonization in the conjunctival sac. J Cataract Refract Surg 2009;35:1609-1613.

7 Hyon JY, Eser I, O’Brien TP: Kill rates of preserved and preservation-free topical 8-methoxy fluoroquinolones against various strains of Staphylococcus. J Cataract Refract Surg 2009;35:1609-1613.

-8 Bhavsar AR, Ip MS, Glassman AR; DRCRnet Study Group, SCORE Study Group: The risk of endophthalmitis following intravitreal triamcinolone injection in the DRCRnet and SCORE clinical trials. Am J Ophthalmol 2007; 144:454-456.

-9 Bhavsar AR, Googe JM Jr, Stockdale CR, Bressler NM, Brucker AJ, Elman MJ, Glassman AR; Diabetic Retinopathy Clinical Research Network: Risk of endophthalmitis after intravitreal drug injection when topical antibiotics are not required: the Diabetic Retinopathy Clinical Research Network Laser-Ranibizumab-Triamcinolone Clinical Trials. Arch Ophthalmol 2009;127:1581-1583.

10 Bhatt SS, Stepien KE, Joshi K: Prophylactic antibiotic use after intravitreal injection: effect on endophthalmitis rate. Retina 2011;31:2032-2036.

11 Ta CN, Egbert PR, Singh K, Shriver EM, Blumenkranz MS, Miño De Kaspar H: Prospective randomized comparison of 3-day versus 1-hour preoperative ofloxacin prophylaxis for cataract surgery. Ophthalmology 2002;109:2036-2040.

12 Moss JM, Sanislo SR, Ta CN: A prospective randomized evaluation of topical gatifloxacin on conjunctival flora in patients undergoing intravitreal injections. Ophthalmology 2009;116:1498-1501.

-13 Jager RD, Aiello LP, Patel SC, Cunningham ET: Risks of intravitreous injection: a comprehensive review. Retina 2004;24:676-698.

14 Moss JM, Nguyen D, Liu YI, Singh K, Montague A, Egbert PR, Kaspar HM, Ta CN: Comparison of one-day versus one-hour application of topical gatifloxacin in eliminating conjunctival bacterial flora. Ophthalmology 2008; 115:2013-2016.

15 Ta CN, He L, Mino de Kaspar H: In vitro antibiotic susceptibility of preoperative normal conjunctival bacteria. Eye (Lond) 2009;23:559-560.

16 Green-Simms AE, Ekdawi NS, Bakri SJ: Survey of intravitreal injection techniques among retinal specialists in the United States. Am J Ophthalmol 2011;151:329-332.

17 Kim SJ, Toma HS, Midha NK, Cherney EF, Recchia FM, Doherty TJ: Antibiotic resistance of conjunctiva and nasopharynx evaluation study: a prospective study of patients undergoing intravitreal injections. Ophthalmology 2010;117:2372-2378.

-18 Han DP, Wisniewski SR, Wilson LA, Barza M, Vine AK, Doft BH, Kelsey SF: Spectrum and susceptibilities of microbiological isolates in the Endophthalmitis Vitrectomy Study. Am J Ophthalmol 1996;122:1-17.

19 Bannerman TL, Rhoden DL, McAllister SK, Miller JM, Wilson LA: The source of coagulase negative staphylococci in the Endophthalmitis Vitrectomy Study. A comparison of eyelid and intraocular isolates using pulsedfield gel electrophoresis. Arch Ophthalmol 1997;115:357-361.

20 Moss JM, Sanislo SR, Ta CN: Antibiotic susceptibility patterns of ocular bacterial flora in patients undergoing intravitreal injections. Ophthalmology 2010;117:2141-2145.

21 Hatch WV, Cernat G, Wong D, Devenyi R, Bell CM: Risk factors for acute endophthalmitis after cataract surgery: a population-based study. Ophthalmology 2009;116:425-430.

22 Fintak DR, Ho AC: Perioperative and operative considerations in diabetics. Ophthalmol Clin North Am 2006; 19:427-434.

23 Holz EH: Reducing endophthalmitis risk factors: where we stand. Am J Ophthalmol 2005;139:1097-1098.

$-24$ Ou JI, Ta CN: Endophthalmitis prophylaxis. Ophthalmol Clin North Am 2006;19:449-456. 